$$
\text { CONF- } 9605186--2
$$

\title{
CALCULATION AND MEASUREMENT OF THE CRITICAL STATE IN SUPERCONDUCTING TAPES
}

\author{
K. L. Telschow and L.S. Koo \\ Idaho National Engineering Laboratory \\ Idaho Falls, ID 83415-2209 \\ PEOEIVED \\ Avg 22 2996 \\ O.S.TI
}

\begin{abstract}
An integral equation approach to solving for the flux front profile in the critical state model is described. Both nonuniform external fields and demagnetizing geometries can be accommodated as long as cylindrical symmetry is preserved. Results for a sphere in a uniform external field and a tape in the field of an external coil parallel to the tape surface are presented.
\end{abstract}

\section{INTRODUCTION}

A method for quantitatively determining the local low field critical current density, $\mathrm{J}_{\mathrm{c}}(\mathrm{H}=0)$, in a noncontacting manner for spatial uniformity inspection is important in the fabrication of high $T_{c}$ superconducting tapes. This can be accomplished by measuring the magnetic response of the sample with small source/pickup probe coils that spatially scan over the tape surface [1]-[3]. Subsequently, the Bean critical state model [4] is used to determine $J_{c}$ from the measured magnetic hysteresis. In this model the induced currents in the sample are either at the critical value or zero, forming a critical state region bounded by a "flux front profile". The response is calculated by summing the fields produced by the currents within this flux front. $\mathrm{J}_{\mathrm{c}}$ can be inferred from the local magnetization measurements. However, demagnetization effects of the 


\section{DISCLAIMER}

Portions of this document may be illegible in electronic image products. Images are produced from the best available original document. 


\section{DISCLAIMER}

This report was prepared as an account of work sponsored by an agency of the United States Government. Neither the United States Government nor any agency thereof, nor any of their employees, makes any warranty, express or implied, or assumes any legal liability or responsibility for the accuracy, completeness, or usefulness of any information, apparatus, product, or process disclosed, or represents that its use would not infringe privately owned rights. Reference herein to any specific commercial product, process, or service by trade name, trademark, manufacturer, or otherwise does not necessarily constitute or imply its endorsement, recommendation, or favoring by the United States Government or any agency thereof. The views and opinions of authors expressed herein do not necessarily state or reflect those of the United States Government or any agency thereof. 
tape/coil geometry are not readily accounted for within the normal extension of the Bean critical state model. This paper describes a method for predicting the flux front profile as a function of the external field that takes into account the demagnetizing effects of the sample surfaces. An integral equation technique is presented that is applicable to sample/probe geometries exhibiting cylindrical symmetry. The net magnetization signal detected by pickup coils is calculated and compared with experimental measurements.

\section{THEORETICAL APPROACH}

Whenever a superconducting material is placed in an applied magnetic field, a region of shielding current is induced. According to the Bean critical state model, the induced current begins at the material boundary next to the applied magnetic field and extends inward, satisfying $\nabla \times \vec{B}=\mu_{0} \vec{J}_{c}$, where $\vec{B}$ is the magnetic induction vector and $\mu_{0}$ is the free-space permeability. This leads to the net magnetic flux density in the material decreasing to zero at the flux front boundary. The region inside this boundary is free of magnetic field, shielded completely by the induced screening currents. This flux front surface inside a superconducting material can be defined as the surface on which the total magnetic field is zero, which results in a vector equation defining the boundary [6]. However, as is proven in [7], it can also be defined as a surface of zero vector potential, $\vec{A}$, where $\nabla \times \vec{A}=\vec{B}$. The use of the vector potential simplifies the calculations since only one component is needed for problems of azimuthal symmetry. For problems with this symmetry, the induced currents inside the material can be modeled as coaxial loops, each carrying a current of constant value, $\mathrm{J}_{\mathrm{c}}$. The vector potential of a single current loop is well known. Hence, the vector potential due to all of the induced currents is a volumetric integral sum of the single loops. The unknown quantities in this integral are the flux front boundary, $\Psi$, which forms part of the integration limit, and the current density, $\mathrm{J}_{\mathrm{c}}$. In addition, the flux front boundary, $\Psi$, is a function of both space and the applied magnetic field. To simplify the calculations, the external applied field is normalized by $\mathrm{J}_{\mathrm{c}}$ and a characteristic length. With this normalizing scheme, the total vector potential, $A_{\text {tot }}$, becomes a dimensionless implicit function of $\Psi(R, \beta)$, a normalized spatial variable, $R$, and the normalized applied field, $\beta$. Then the total vector potential is given by $A_{t o t}=A_{\beta}-A_{J_{c}}$, where $A_{\beta}$ and $A_{J_{c}}$ are the vector potentials due to the external field, $\beta$, and the induced critical currents, $J_{c}$. The minus sign results from the shielding effect. For a given $\beta$, the position of the flux front surface is determined by finding spatial points where the total vector potential is zero. This is a difficult problem in general, but can be simplified by reduction to a single integral equation as follows. In general $\beta$ is a function of time. This technique deals only with the quasi-stationary states of the critical state. The time scale for changes in the external field is typically very much longer than that exhibited by flux line motion, so the model always assumes a sequence of stationary states uniquely defined by the history and present value of the external field. Changes in the external 
field then produce a corresponding change in the flux line profile position, but at all times the total vector potential on this profile is zero. Therefore, a requirement for determining the flux penetration profile is that

$$
\frac{\partial A_{\text {tot }}(\Psi(R, \beta), R)}{\partial \beta}=F\left(\Psi(R, \beta), \frac{\partial \Psi(R, \beta)}{\partial \beta}\right)=0 .
$$

Equation (1) is often a nonlinear integral equation. As suggested by the critical state model, when the external field, $\beta$, is initially turned on, flux enters the superconducting material from the surface. Thus the initial profile, $\Psi(R, \beta=0)$, is the material surface. Substitution of the known surface profile into (1) yields a linear integral equation of the first kind for the unknown derivative of $\Psi$. The algorithm analyzed by Gold [8] is used to resolve (1). As the external field is increased to $\beta=\Delta \beta$, the new flux front profile is approximated by

$$
\Psi(R, \beta=\Delta \beta)=\Psi(R, \beta=0)+\frac{\partial \Psi(R, \beta=0)}{\partial \beta} \cdot \Delta \beta
$$

This approximation is acceptable as long as the increment $\Delta \beta$ is sufficiently small. With this approximation the flux front profile at $\beta=\Delta \beta$ is known but its derivative is yet to be determined. This is the same situation as at the beginning when $\beta=0$. The above procedure is repeated and the new $\beta$ value determined. This methodology results in a progressive incremental numerical scheme in $\beta$ and an iterative procedure for resolving (1) for each $\beta$. Once the flux front profile's dependence on the external field for the zero-field cooled (ZFC) case is known, then the response of the sample to a complete cycle of changes in the external field can be readily calculated, knowing $\mathrm{M}_{\mathrm{ZFC}}(\beta)$, as follows:

$$
\mathrm{M}_{ \pm}(\beta)= \pm \mathrm{M}_{\mathrm{ZFC}}\left(\beta_{\max }\right) \mp 2 \mathrm{M}_{\mathrm{ZFC}}\left(\frac{\beta_{\max } \mp \beta}{2}\right)
$$

where ( + ) means decreasing $\beta_{\max }>\beta>-\beta_{\max }$ and (-) increasing $-\beta_{\max } \rightarrow \beta \rightarrow+\beta_{\max }$ in the external field. Examples of this calculation for a spherical sample in a uniform external field and an infinite plate in the field of an external single loop source coil are described below.

\section{SPHERE IN A UNIFORM EXTERNAL FIELD}

An example of a cylindrically symmetric application is that of a sphere in a uniform external field. Let the radius of the sphere be $\mathrm{r}_{0}$ and the flux front surface be described by $\rho(z, \beta)$ where the external field dependence is included in the normalization parameter $\beta=\mathrm{H}_{\mathrm{z}}^{\text {ext }} /\left(\mathrm{r}_{0} \mathrm{~J}_{\mathrm{c}}\right)$. The 
configuration is depicted in Fig. 1. Also shown in this figure are two flux fronts trapped inside the sphere as a result of extending the external field to a maximum value below the full penetration value. The total normalized vector potential at $(\mathrm{r}, \mathrm{z})$ is given below [7], where all lengths are normalized to the sphere radius $R=r_{1} / r_{0} ; Z=z / r_{0} ; \Psi=r / r_{0} ;$ etc. and $\mu_{0} \mathrm{a}_{\phi}\left(R, Z ; R^{\prime}, Z^{\prime}\right)$ is the vector potential at $(R, Z)$ due to a single current loop at ( $\left.R^{\prime}, Z^{\prime}\right)$

$$
A_{\phi}(R, Z, \beta)=\frac{\beta R}{2}-\int_{-1}^{1} d Z^{\prime} \int_{\Psi\left(Z^{\prime}, \beta\right)}^{\sqrt{1-Z^{\prime 2}}} d R^{\prime} a_{\phi}\left(R, Z ; R^{\prime}, Z^{\prime}\right)
$$

A single integral equation describing the flux front surface for a sphere results

$$
\int_{-1}^{1} d Z^{\prime} a_{\phi}\left(\Psi(Z, \beta), Z ; \Psi\left(Z^{\prime}, \beta\right), Z^{\prime}\right) \frac{-\partial \Psi\left(Z^{\prime}, \beta\right)}{\partial \beta}=\frac{\Psi(Z, \beta)}{2} .
$$

The intial profile of the flux front is $\Psi(Z, \beta=0)=\sqrt{1-Z^{2}}$. All flux fronts for the ZFC case are obtained by following the aforementioned incremental iteration procedure. The full field penetration value obtained yields a normalized external field $\beta^{*}=0.789$. A value of $\pi / 4$ is found analytically for a completely filled sphere. The numerical and analytical values agree to within $0.5 \%$ [7] which is consistent with the expected accuracy of the calculation procedure used. The subsequent magnetization hysteresis curve, $M(\beta)$, for the sphere due to a complete cycle of change in the external field can be obtained through equation (3). The results are given in Fig. 2 , normalized by the full penetration magnetization $\mathrm{M}^{*}=3 \mathrm{pr}_{0} \mathrm{~J}_{\mathrm{c}} / 32$.

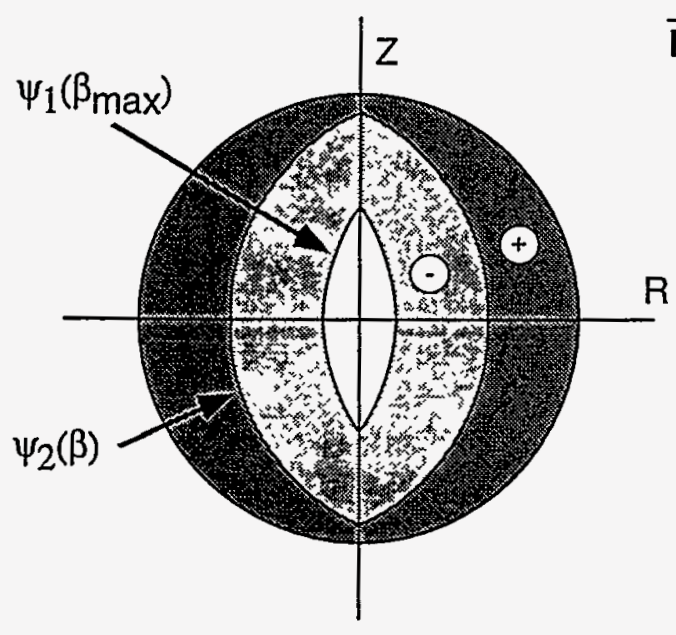

Fig. 1. Flux front profiles for a sphere.
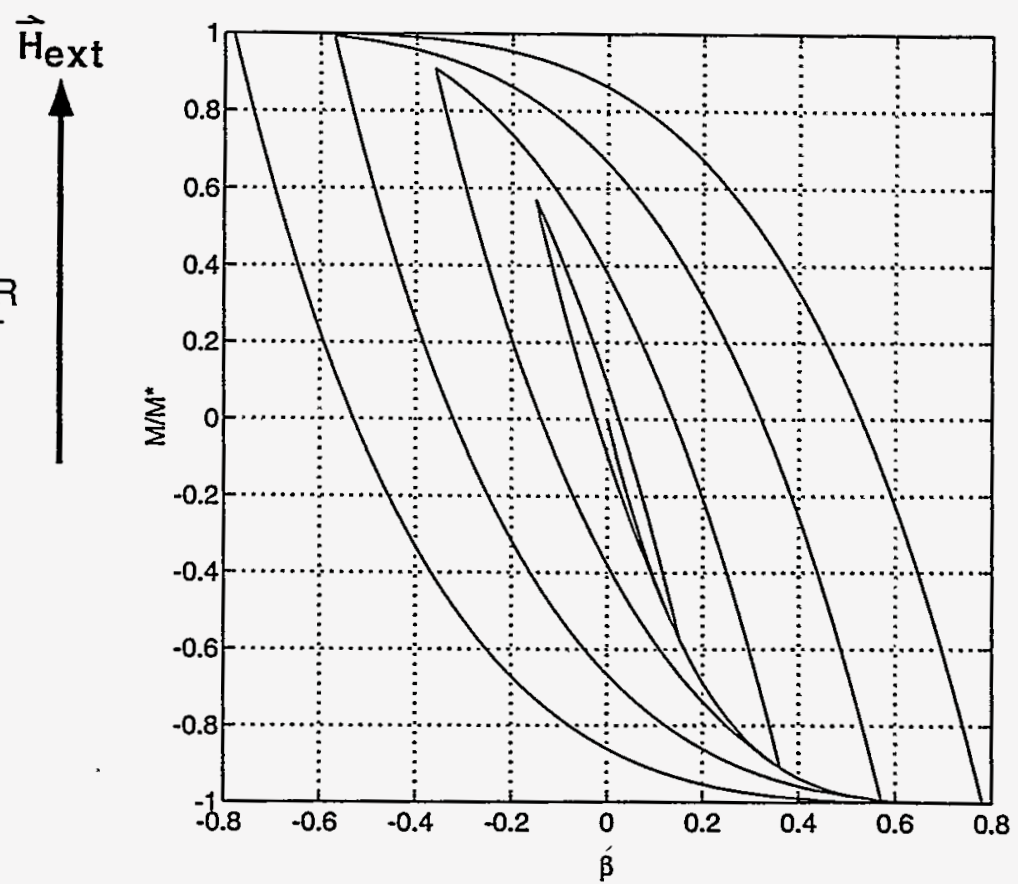

Fig. 2. Magnetization hysteresis of the sphere for various limiting fields. 


\section{TAPE GEOMETRY}

A single circular coil above a superconducting infinite plate of thickness $D$ is shown in Fig. 3. All geometrical lengths are normalized by the coil radius, $\mathrm{r}_{\mathrm{c}}$. The driving coil is placed at $\left(1, \mathrm{Z}_{\mathrm{C}}\right)$. The

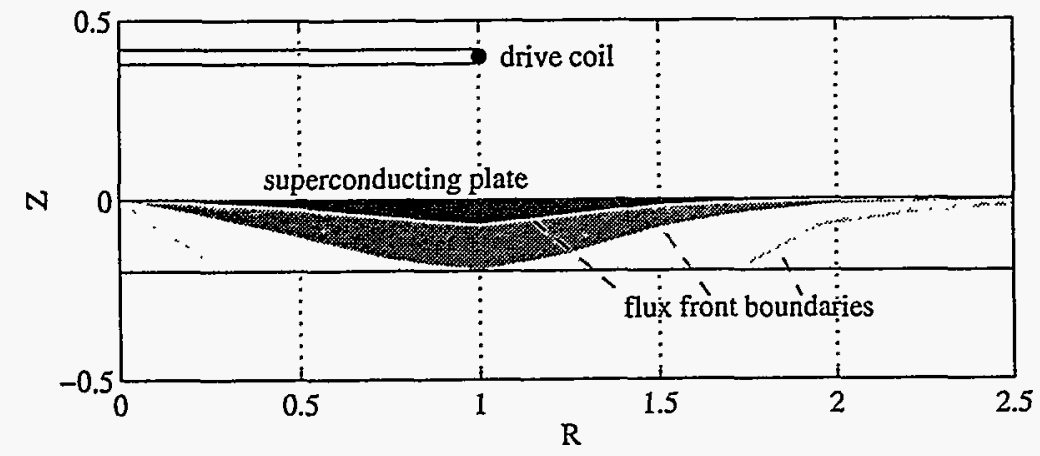

Fig. 3. Geometry for flux penetration in a plate for ZFC case with $\beta=0.1,0.365$ and 0.865 . normalized external field is $\beta=\mathrm{I} / \mathrm{J}_{\mathrm{C}}$ $\mathrm{rc}^{2}$, where $\mathrm{I}$ is the current in the driving coil. The flux front profiles for the $\mathrm{ZFC}$ case are also shown in Fig. 3. At $\beta=0, \Psi=0$ coincides with the plate top surface. As $\beta$ is increased to the next value, $\Psi$ shows a profile that penetrates ..deepest directly under the driving coil. This deepest value of $\Psi$ reaches the plate bottom edge at $\beta^{*}$ $=0.365$, for $\mathrm{D}=-0.2$. Beyond this

value, the flux front is broken up into two sections: an inner section, $\Psi_{1}$, which approaches $\mathrm{R}=0$, and an outer section, $\Psi_{2}$, which approaches $R=\infty$ as $\beta$ increases. The intersections between $\Psi_{1}$, $\Psi_{2}$ and the bottom surface, $Z=D$, are denoted as $R_{1}$ and $R_{2}$, respectively. Before the bifurcation of the flux front surface, the total vector potential amplitude (normalized by $\mu_{0} J_{c} r_{c}^{2}$ ) is

$$
A_{\text {tot }}(R, Z)=\beta a_{\phi}\left(R, Z ; 1, Z_{c}\right)-\int_{0}^{\infty} d R^{\prime} \int_{\Psi\left(R^{\prime}, \beta\right)}^{0} d Z^{\prime} a_{\phi}\left(R, Z ; R^{\prime}, Z^{\prime}\right)
$$

where $\mu_{0} a_{\phi}\left(R, Z ; R^{\prime}, Z^{\prime}\right)$ is the vector potential at $(R, Z)$ due to a unit current loop at ( $\left.R^{\prime}, Z^{\prime}\right)$. A single integral equation results for the flux front surface

$$
\int_{0}^{\infty} d R^{\prime} a_{\phi}\left(R, \Psi(R, \beta) ; R^{\prime}, \Psi\left(R^{\prime}, \beta\right)\right) \frac{-\partial \Psi\left(R^{\prime}, \beta\right)}{\partial \beta}=a_{\phi}\left(R, \Psi(R, \beta) ; 1, Z_{c}\right) .
$$

The initial profile of the flux front is $\Psi(R, \beta=0)=0$. As $\beta$ is increased beyond $\beta^{*}$, the flux front surface $\Psi$ will become two separate surfaces, $\Psi_{1}$ and $\Psi_{2}$. The total vector potential becomes 


$$
\begin{aligned}
A_{\text {tot }}(R, Z)= & \beta a_{\phi}\left(R, Z ; 1, Z_{c}\right)-\int_{0}^{R_{1}(\beta)} d R^{\prime} \int_{\Psi_{1}\left(R^{\prime}, \beta\right)}^{0} d Z^{\prime} a_{\phi}\left(R, Z ; R^{\prime}, Z^{\prime}\right) \\
& -\int_{R_{1}(\beta)}^{R_{2}(\beta)} d R^{\prime} \int_{D}^{0} d Z^{\prime} a_{\phi}\left(R, Z ; R^{\prime}, Z^{\prime}\right)-\int_{R_{2}(\beta)}^{\infty} d R^{\prime} \int_{\Psi_{2}\left(R^{\prime}, \beta\right)}^{0} d Z^{\prime} a_{\phi}\left(R, Z^{\prime} ; R^{\prime}, Z^{\prime}\right)
\end{aligned} .
$$

With $\Psi_{1}^{\prime}$ and $\Psi_{2}^{\prime}$ denoted as $\Psi_{1}\left(R^{\prime}, \beta\right)$ and $\Psi_{2}\left(R^{\prime}, \beta\right)$, two coupled equations result [9]

$$
\begin{aligned}
& \int_{0}^{R_{1}(\beta)} d R^{\prime} a_{\phi}\left(R, \Psi_{1} ; R^{\prime}, \Psi_{1}^{\prime}\right) \cdot \frac{-\partial \Psi_{1}^{\prime}}{\partial \beta}+\int_{R_{2}(\beta)}^{\infty} d R^{\prime} a_{\phi}\left(R, \Psi_{1} ; R^{\prime}, \Psi_{2}^{\prime}\right) \cdot \frac{-\partial \Psi_{2}^{\prime}}{\partial \beta}=a_{\phi}\left(R, \Psi_{1} ; 1, Z_{c}\right) \\
& \int_{0}^{R_{1}(\beta)} d R^{\prime} a_{\phi}\left(R, \Psi_{2} ; R^{\prime}, \Psi_{1}^{\prime}\right) \cdot \frac{-\partial \Psi_{1}^{\prime}}{\partial \beta}+\int_{R_{2}(\beta)}^{\infty} d R^{\prime} a_{\phi}\left(R, \Psi_{2} ; R^{\prime}, \Psi_{2}^{\prime}\right) \cdot \frac{-\partial \Psi_{2}^{\prime}}{\partial \beta}=a_{\phi}\left(R, \Psi_{2} ; 1, Z_{c}\right) .
\end{aligned}
$$

These two equations couple both flux fronts together. In the first equation, the observation points are on $\Psi_{1}$, while in the second equation the observation points are on $\Psi_{2}$. These two equations, expressed in matrix format, are

$\left[\begin{array}{ll}K_{11} & K_{12} \\ K_{21} & K_{22}\end{array}\right]\left(\begin{array}{l}f_{1} \\ f_{2}\end{array}\right)=\left(\begin{array}{l}a_{1} \\ a_{2}\end{array}\right)$ where $\quad K_{12} f_{2}=\int_{R_{2}(\beta)}^{\infty} d R^{\prime} a_{\phi}\left(R, \Psi_{1} ; R^{\prime}, \Psi_{2}^{\prime}\right) \cdot \frac{-\partial \Psi^{\prime}}{\partial \beta}$, etc.
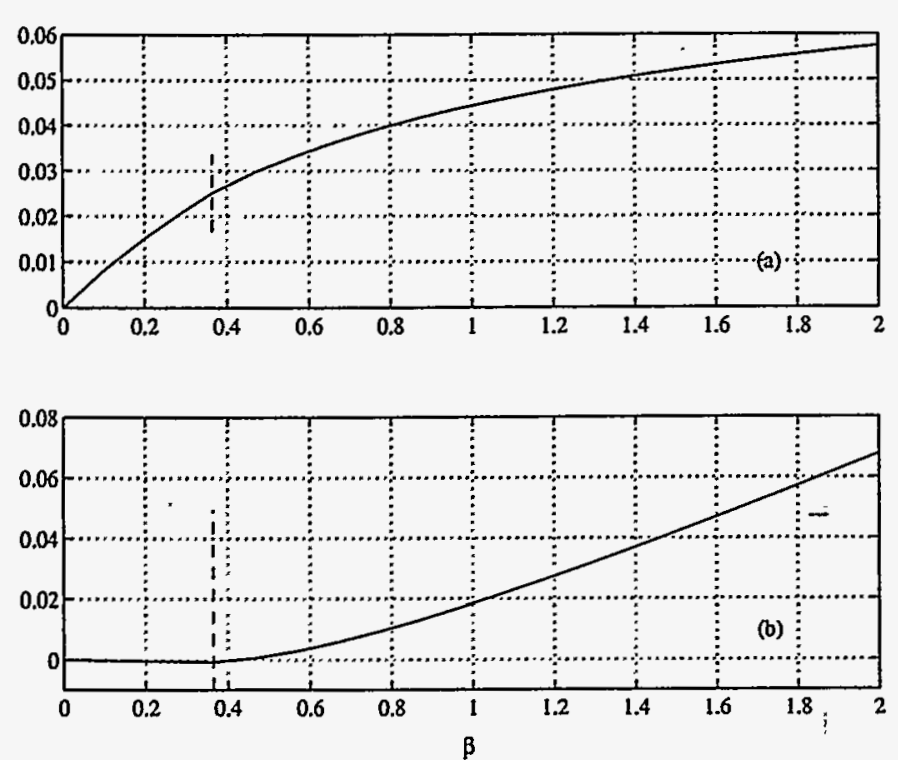

Fig. 4: $A_{J_{c}}$ for a coil above the plate (a) and $A_{t o t}$ for a coil beneath the plate (b).
The incremental and iterative procedures were employed to resolve the two separate flux fronts simultaneously. Two simulated measurement examples are given in Figs. $4 \mathrm{a}$ and $4 \mathrm{~b}$. In these examples, $\mathrm{r}_{\mathrm{c}}=0.5 \mathrm{~mm}, Z_{\mathrm{c}}=0.4$, $\mathrm{D}=-0.2$. In Fig. $4 \mathrm{a}$, the normalized screening current vector potential amplitude, $\mathrm{A}_{\mathrm{J}}$, at the driving coil position $(1,0.4)$ is given, simulating the measurement results found above the sample with a top balanced coil. In Fig. $4 \mathrm{~b}$, the normalized total vector potential amplitude, $A_{\text {tot }}$ is given at $(1,-0.6)$, simulating the measurement results found beneath the sample with a single unbalanced coil. The vertical dashed line signifies the value $\beta^{*}$ where the flux front fully penetrates to the plate bottom surface. 


\section{EXPERIMENTAL MEASUREMENTS}

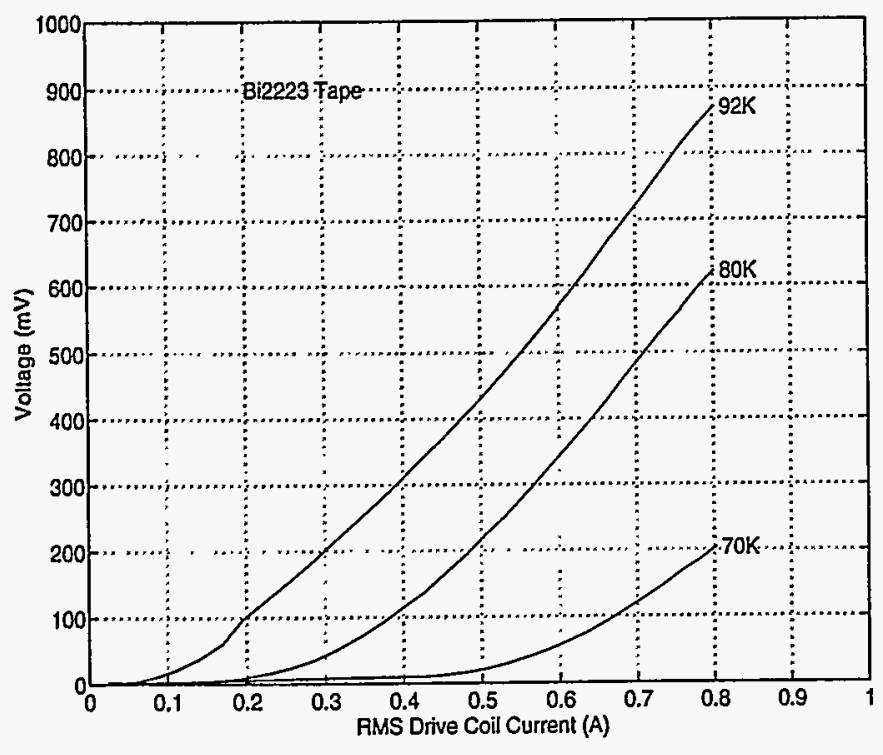

Fig. 5. Measured bottom coil signal amplitudes for different temperatures.
The measurement geometry is that of a single layer superconductor plate deposited between two outside silver layers. Tape samples of $\mathrm{Pb}-\mathrm{BiSrCaCuO}$ (2223 phase) produced by the powder-intube method [3],[5] were measured with a small probe source coil (13 turns of \#36 copper wire, $1 \mathrm{~mm}$ inside diameter). Balanced opposing pickup coils (5 turns each of \#36) were wound over the source coil, producing a small source/pickup probe that could be scanned over the sample surface in a liquid nitrogen bath. An additional pickup coil (5 turns \#36 copper wire on $1 \mathrm{~mm}$ diameter) was positioned below the tape sample. Lift-off distances for both top and bottom coils were about $0.7 \mathrm{~mm}$.

Fig. 5 shows the measured results from the bottom coil at one position on the tape for three different temperatures. These results, which are qualitatively similar to the theoretical results of Fig. $4 \mathrm{~b}$, represent the magnetization due only to the induced screening currents within the flux front. The shielding effect is clearly visible and, by extrapolation, a unique point corresponding
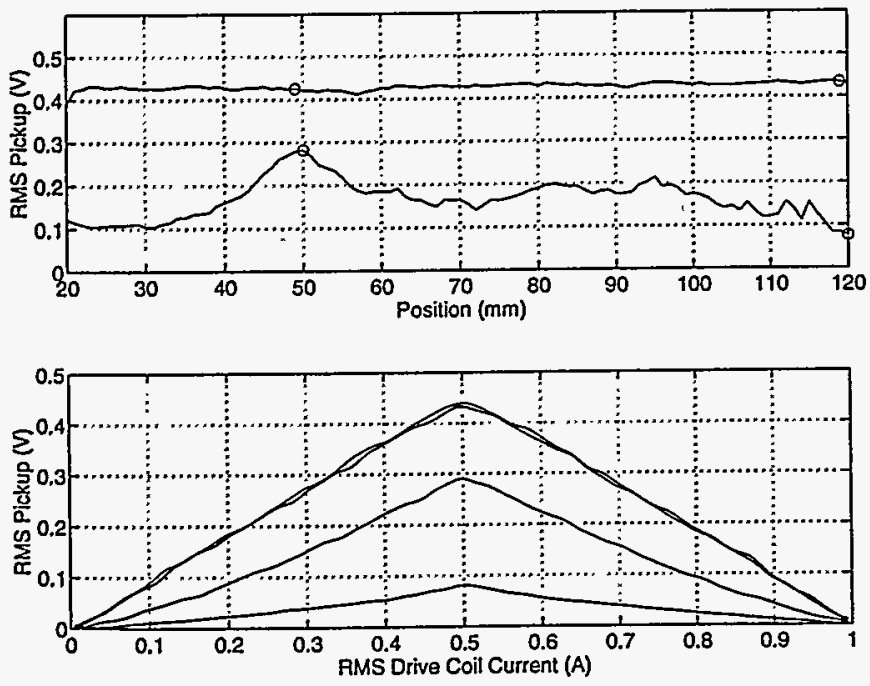

Figure 6. Maximum signal along a tape (top) and corresponding RMS signal amplitudes as a function of the source coil current. to full plate penetration can be estimated. The local critical current density can then be determined from the corresponding value of $\beta$.

Tapes produced by the "powder in tube" method exhibit thickness variations as well as inhomogeneity in the superconducting layer.

Nevertheless, the above approach can be used to provide a measure of uniformity and local critical current density. Figure 6 shows typical results for a scan of the small probe along a tape [5], produced by the powder in tube method.

Significant variations are observed due to local microstructural inhomogeneities. 
Detailed information like that shown in figure 6 is helpful in locating problems associated with intergrain connections and thickness or porosity defects.

\section{CONCLUSION}

A method has been outlined for calculating the flux front profile for a superconducting sample in either a uniform or nonuniform applied magnetic field possessing cylindrical symmetry. This technique extends the Bean critical state model by fully accounting for demagnetizing effects. The method relies upon finding the flux front penetration profile found by resolving a linear integral equation of the first kind. Measurement-induced voltages and the entire hysteresis loop response can be found by extension of the ZFC magnetization response with a changing external field. Other measured quantities relating to the critical state can be calculated directly from the hysteresis loop if the time dependence of the external field is known.

\section{ACKNOWLEDGMENT}

The authors thank M. T. Lanagan of Argonne National laboratory for the tape samples used and K. K. Haulenbeek and F. Bruneel for help in obtaining the measurement results. This work was supported by the U.S. Department of Energy, Office of Basic Energy Sciences, under DOE Idaho Operations Office Contract DE-AC07-94ID13223.

\section{REFERENCES}

[1] A. M. Campbell and J. E. Evetts, Critical Currents in Superconductors (Taylor \& Francis Ltd., London, 1972).

[2] K. L. Telschow and T. K. O'Brien, Appl. Phys. Lett. 59, 730 (1991).

[3] K. L. Telschow, T. K. O'Brien, M. T. Lanagan and D. Y. Kaufman, IEEE Trans. Magn., vol. $\underline{3}$ (1) part III, 1643-1646, March 1993.

[4] C. P. Bean, Phys. Rev. Lett. 8, 250 (1962) and Rev. Mod. Phys. 36,31 (1964).

[5] Samples were provided by M. T. Lanagan at Argonne National Laboratory.

[6] R. Navarro and L. J. Campbell, Phys. Rev. B 44, 10146 (1991).

[7] K. L. Telschow and L. S. Koo, Phys. Rev. B 50 (10), 6923 (1994).

[8] R. Gold, ANL-6984, Argonne National Laboratory, December 1964.

[9] L. S. Koo and K. L. Telschow, Phys. Rev. B 53, 8743 (1996). 